\title{
Stochastic Carrier Dynamics in Semiconductor Superlattices
}

T.M. Fromhold ${ }^{a}$, S. Bujkiewicz ${ }^{a, *}$, A. Patanì $\grave{1}^{a}$ S.P. Stapleton ${ }^{a}$, D. Sherwood ${ }^{a}$, D. Fowler ${ }^{a}$, J. CoOper $^{a}$,

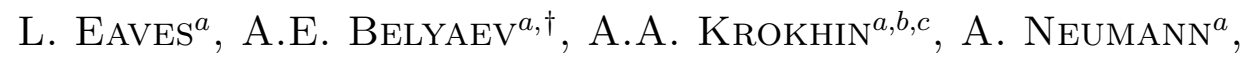
P.B. Wilkinson ${ }^{a}$, N.S. SANKESHWAR ${ }^{a \ddagger}$, M. HENINI $^{a}$ AND F.W. SHEARD ${ }^{a}$

${ }^{a}$ School of Physics and Astronomy, University of Nottingham Nottingham NG7 2RD, UK

${ }^{b}$ Instituto de Fisica, Universidad Autonoma de Puebla, Puebla 72570, Mexico

${ }^{c}$ Center for Nonlinear Science, University of North Texas P.O. Box 5368, Denton, Texas 762203, USA

\begin{abstract}
We explore a new regime of hot carrier dynamics, in which electrons in a superlattice miniband exhibit a unique type of stochastic motion when a magnetic field is tilted at an angle $\theta$ to the superlattice axis. Remarkably, the dynamics of a miniband electron in a tilted magnetic field reduce to a one-dimensional simple harmonic oscillator, of angular frequency $\omega_{\mathrm{C}} \cos \theta$, where $\omega_{\mathrm{C}}$ is the cyclotron frequency, driven by a time-dependent plane wave whose angular frequency equals the Bloch frequency $\omega_{\mathrm{B}}$. At bias voltages for which $\omega_{\mathrm{B}}=n \omega_{\mathrm{C}} \cos \theta$, where $n$ is an integer, the electron orbits change from localised Bloch-like trajectories to unbounded stochastic orbits, which diffuse rapidly through intricate web patterns in phase space. To quantify how these webs affect electron transport, we make drift-diffusion calculations of the current-voltage curves including the effects of space-charge build up. When the magnetic field is tilted, our simulations reveal a large resonant peak, which originates from stochastic delocalisation of the electron orbits. We show that the corresponding quantised eigenstates change discontinuously from a highly localised character when the system is off resonance to a fully delocalised form when the resonance condition is satisfied.
\end{abstract}

PACS numbers: 73.20.At, 73.21.Cd

*Permanent address: Institute of Physics, Wrocław University of Technology, Wybrzeże Wyspiańskiego 27, 50-370 Wrocław, Poland.

${ }^{\dagger}$ Permanent address: Institute of Semiconductor Physics, NASU, Kiev 03028, Ukraine.

${ }^{\ddagger}$ Permanent address: Department of Physics, University of Karnatak, Dharwad, India. 


\section{Introduction}

In semiconductor physics, chaotic electron transport has been explored using a variety of two-dimensional billiard and antidot barrier structures [1-12], in superlattices (SLs) [13-15], and in resonant tunnelling diodes containing a wide quantum well with a tilted magnetic field [1, 16-18]. Despite the diversity of these experiments, they all involve systems in which the transition to chaos occurs by the gradual and progressive destruction of stable orbits in response to an increasing perturbation. But there is also a much rarer type of chaos, known as non-KAM dynamics [19-21], which switches on and off abruptly when the perturbation reaches certain critical values. The theory of these so-called "non-KAM" systems is of great interest due to applications in plasma physics, tokamak fusion, turbulent fluid dynamics, ion traps, and quasicrystals [19]. But the quantum properties of such systems are poorly understood, and, to our knowledge, have never been investigated in experiment. In this paper, we show that electrons in a SL miniband with a tilted magnetic field provide an experimentally-accessible non-KAM system and, moreover, that this unusual type of chaotic dynamics can have a pronounced effect on the current-voltage characteristics of real devices.

To demonstrate this effect, we consider a novel type of GaAs/InAs/AlAs SL structure [22]. The unusual feature is that at the centre of each GaAs quantum well, there is an InAs monolayer, which produces a notch in the conduction band edge [22]. Since this notch coincides with the antinode in the ground state wave function of the quantum well, its effect is to lower the energy of the first miniband down to the bottom of the quantum well. This ensures that the edge of the miniband lies close to the Fermi level in the emitter contact, which (a) facilitates electron injection into the miniband, (b) ensures that the miniband gap energy has an extremely large value of $240 \mathrm{meV}$. This suppresses inter-band tunnelling [22] and thereby ensures that a single band transport picture is appropriate.

\section{Semiclassical dynamics, phase space structure and quantised energy eigenstates}

We consider electron motion in the first miniband using a tight-binding approximation for the energy versus crystal momentum dispersion relation corresponding to motion along the SL $(x-)$ axis. The SL period $a=8 \mathrm{~nm}$, the miniband width $\Delta=13.5 \mathrm{meV}$ and the density of $n$-type doping in the SL layers is $3 \times 10^{16} \mathrm{~cm}^{-3}[22]$. To investigate the dynamics of electrons in the miniband, we used the semiclassical equations of motion $\boldsymbol{v}=\partial E(\boldsymbol{p}) / \partial \boldsymbol{p}$, and $\mathrm{d} \boldsymbol{p} / \mathrm{d} t=$ $-e[\boldsymbol{F}+(\boldsymbol{v} \times \boldsymbol{B})]$, where $\boldsymbol{v}$ and $\boldsymbol{p}$ are, respectively, the electron's velocity and crystal momentum, the electron energy $E(\boldsymbol{p})=\Delta\left[1-\cos \left(p_{x} a / \hbar\right)\right] / 2+\left(p_{y}^{2}+p_{z}^{2}\right) / 2 m^{*}$, where $m^{*}$ is the effective mass, $e$ is the magnitude of the electronic charge, $\boldsymbol{F}$ is the electric field produced by the applied voltage $V$, and $\boldsymbol{B}$ is the applied magnetic field. We consider motion in a tilted magnetic field which lies in the $x-z$ plane at an angle $\theta$ to the SL axis (Fig. 1, inset). 


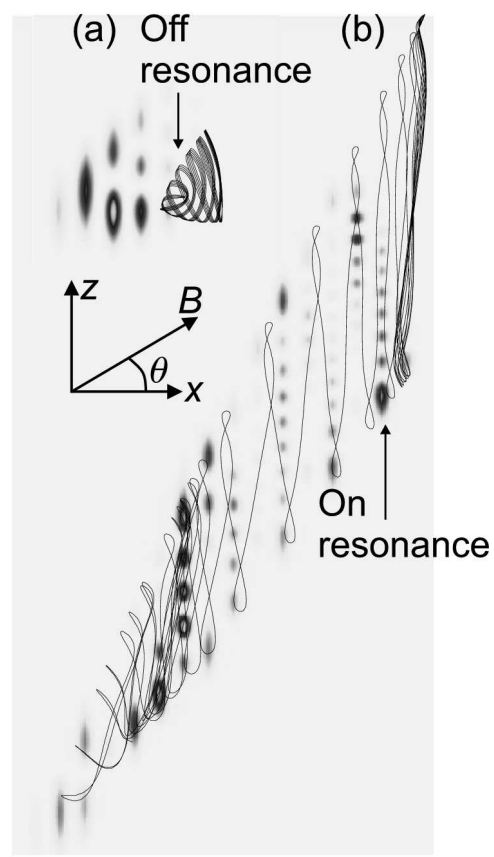

Fig. 1. Electron orbits in the $x-z$ plane (axes inset) superimposed on probability density plots (black $=$ high) of quantised eigenstates calculated for the SL. (a) A highly localised orbit (offset for clarity) off resonance at $\theta=30^{\circ}$. (b) An unbounded stochastic orbit on the $n=1$ resonance at $\theta=60^{\circ} . B=11 \mathrm{~T}$.

To our surprise, we found that the dynamics of a miniband electron in a tilted magnetic field are exactly equivalent to a well-known one-dimensional (1D) system, which exhibits non-KAM chaos and has a rather beautiful phase space. The reason for this is that the in-plane component of electron momentum $p_{z}$ satisfies the equation of motion

$$
\frac{\mathrm{d}^{2} p_{z}}{\mathrm{~d} t^{2}}+\left(\omega_{\mathrm{C}} \cos \theta\right)^{2} p_{z}=\left(C B^{2} \Delta \sin 2 \theta\right) \sin \left(K p_{z}-\omega_{\mathrm{B}} t\right)
$$

which corresponds to a one-dimensional simple harmonic oscillator driven by a time $(t-)$ dependent plane wave [21]. In this equation, $C$ is a constant and $K=-a \tan \theta / \hbar$. The angular frequency of the plane wave equals the Bloch frequency $\omega_{\mathrm{B}}=e F a / \hbar$ and the natural frequency of the harmonic oscillator equals the cyclotron frequency $\omega_{\mathrm{C}}$ corresponding to the magnetic field component along the $x$-axis. We emphasise that electron motion in the superlattice depends only on this $1 \mathrm{D}$ equation of motion because its solution $p_{z}$ uniquely determines all of the other dynamical variables and, in particular, the electron orbits in real space [21]. Consequently, the semiclassical dynamics of a miniband electron in a tilted magnetic field reduces to a harmonic oscillator driven by a plane wave, which is a well-known non-KAM chaotic system [19-21]. 
We now consider the semiclassical electron orbits obtained by solving Eq. (1) numerically using a 4th-order Runge-Kutta algorithm. When $\theta=0^{\circ}$, the plane wave on the right-hand-side of Eq. (1) has zero amplitude, which means that the motion along the $x$ - and $z$-directions is separable. Consequently, the electrons undergo cyclotron motion about the magnetic field axis and Bloch oscillations along the $x$-direction, with a peak-to-peak amplitude of $\Delta / e F$. When the magnetic field is tilted, the electron orbits generally remain localised as shown in Fig. 1a. However, at discrete voltages for which $\omega_{\mathrm{B}}=n \omega_{\mathrm{C}} \cos \theta$, where $n$ is an integer, the electron orbits change from localised Bloch-like trajectories to unbounded stochastic orbits (Fig. 1b). The quantised eigenstates also change discontinuously from a highly localised character when the system is off resonance (Fig. 1a) to a fully delocalised form when the resonance condition is satisfied (Fig. 1b).

The resonant delocalisation of the electron trajectories can be understood by considering the phase space of the underlying driven harmonic oscillator. Figure $2 \mathrm{a}$ shows a Poincaré section (slice through the phase space) calculated from several electron orbits, with the same total energy, at $\theta=0^{\circ}$. The section is constructed by plotting $p_{z}$ and $\mathrm{d} p_{z} / \mathrm{d} t \propto p_{y}$ at discrete equally-spaced times along the electron paths [21]. The points in the Poincaré section lie on concentric rings because the electrons undergo simple harmonic cyclotron motion about the magnetic field axis. Rings with larger radii correspond to orbits with higher cyclotron energy associated with motion in the $y-z$ plane. But since all of the orbits used to construct the section have the same total energy, those with higher cyclotron energy must be located further along the $x$-axis where the electrostatic potential energy is lower. In fact, it is easy to show by considering the electron's total energy that the radius of each ring is $\propto \sqrt{x}$. Since each electron orbit is confined to a single ring in the Poincaré section, it is localised in a particular region of the $x$-axis, where it performs Bloch oscillations and cannot propagate through the SL unless it scatters from one orbit to another.

When the magnetic field is tilted, the phase space changes dramatically whenever $\omega_{\mathrm{B}}=n \omega_{\mathrm{C}} \cos \theta$, that is at discrete bias voltages. When this resonance condition is satisfied, the electron trajectories map out intricate web patterns, known as stochastic webs, in the phase space of the underlying driven harmonic oscillator. Figure 2b shows the stochastic web corresponding to the $n=3$ resonance, constructed by plotting $p_{y}$ and $p_{z}$ at discrete equally-spaced times. The filaments of the web extend from the chaotic sea which forms at the centre of the Poincaré section, where the electrons have low energy and momentum and are therefore strongly perturbed by the plane wave. In effect, the radial filaments link together the harmonic-oscillator-like rings that are isolated from one another when $\theta=0^{\circ}$, and this frees the electrons. Of crucial importance for understanding the significance of the stochastic web is that, just as for $\theta=0^{\circ}$, the distance of each point from the centre of the web is proportional to $\sqrt{x}$. So as the electron travels further through the SL, it produces points further from the centre of the section. 


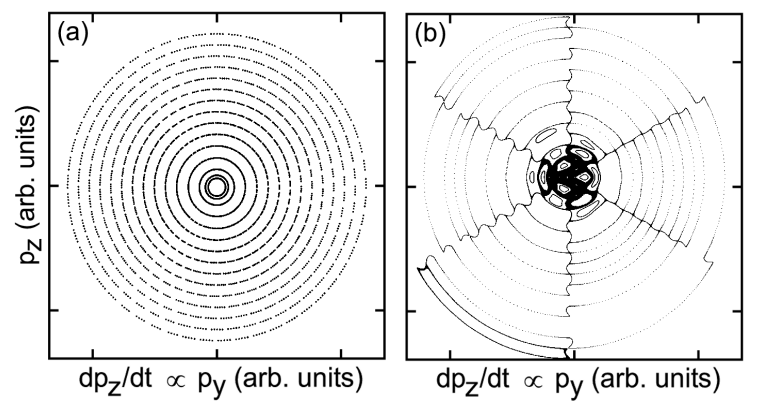

Fig. 2. Poincaré sections (slices through classical phase space) showing momentum components $p_{y}, p_{z}$ in the plane of the SL layers for (a) $\theta=0^{\circ}$, (b) $\theta=60^{\circ}$ with $F$ chosen so that $\omega_{\mathrm{B}}=3 \omega_{\mathrm{C}} \cos \theta$. In (b), the phase space is threaded by an infinite stochastic web (only the central part is shown here) whose chaotic filaments delocalise the electron orbits (see Fig. 1b). $B=11 \mathrm{~T}$.

Electrons are driven out along the radial filaments of the web by the resonant absorption of energy from the plane wave, and so progress rapidly through the SL. Physically, as the electron moves along the SL, the potential energy that it gains from the electric field is transferred into kinetic energy associated with motion in the $y-z$ plane by the tilted magnetic field. Consequently, the electron moves rapidly away from the centre of the web in $\left(p_{y}, p_{z}\right)$ space. Since the web extends to infinity, though only the central part is shown in Fig. 2b, the electron orbits are unbounded and should therefore produce a large direct current. However, moving the voltage infinitesimally off resonance destroys the stochastic web by breaking the radial filaments. This localises the electron orbits and suppresses the current flow. Consequently, non-KAM chaos provides a new and, in principle, extremely sensitive way to modulate the SL conductivity. Indeed, in the absence of scattering, stochastic web formation would change the SL from an insulator to a conductor at discrete bias voltages.

\section{Drift-velocity versus electric field curves}

To quantify the effect of the stochastic webs on the electrical properties of the $\mathrm{SL}$, we calculated the electron drift velocity $v_{\mathrm{d}}$ using the classical kinetic formula

$$
v_{\mathrm{d}}(F)=\frac{1}{\tau} \sum \int_{0}^{\infty} v_{x}(t) \exp (-t / \tau) \mathrm{d} t
$$

in which the summation is over all the possible starting velocities consistent with the electron injection energy. We included electron scattering in our calculations within the relaxation time approximation, using a scattering time $\tau=200 \mathrm{fs}$ determined from experiment [22].

When the magnetic field is applied along the SL axis, the $v_{\mathrm{d}}(F)$ curve (lower trace in Fig. 3) obtained from the regular Bloch orbits is identical to that predicted by the famous Esaki-Tsu model [23]. When the magnetic field is applied 


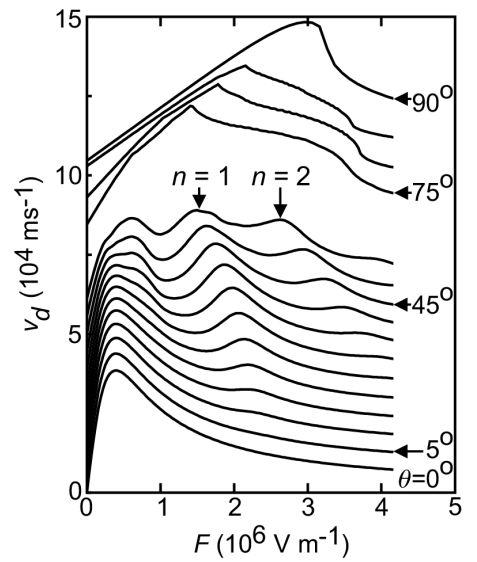

Fig. 3. Drift velocity versus electric field curves calculated for the SL with $B=11 \mathrm{~T}$ and (from bottom to top) $\theta=0^{\circ}, 5^{\circ}, 10^{\circ}, 15^{\circ}, 20^{\circ}, 25^{\circ}, 30^{\circ}, 35^{\circ}, 40^{\circ}, 45^{\circ}, 50^{\circ}$, $55^{\circ}, 75^{\circ}, 80^{\circ}, 85^{\circ}$, and $90^{\circ}$. Arrows indicate resonant peaks originating from electron delocalisation when $n=1$ and 2 .

perpendicular to the SL axis, the electron orbits remain regular, but the peak drift velocity occurs at a higher electric field (top trace in Fig. 3). Hutchinson and co-workers have shown previously that this shift occurs because the magnetic field bends the electron trajectories, thereby reducing the average velocity along the SL axis [24]. Therefore the electric field must be increased in order to restore this average velocity to its maximum value.

When the magnetic field is tilted, the drift velocity curves in Fig. 3 reveal resonant peaks (arrowed) when $\omega_{\mathrm{B}} /\left(\omega_{\mathrm{C}} \cos \theta\right)$ equals 1 and 2 , that is when the electron orbits are delocalised. Stochastic web formation therefore modulates the $v_{\mathrm{d}}(F)$ curves with a series of additional resonant peaks, whose positions can be tuned by simply changing the orientation of $\boldsymbol{B}$.

\section{Current-voltage and conductance-voltage curves}

To determine how the stochastic-web-induced resonances show up in an experimentally-measurable transport parameter, we used the $v_{\mathrm{d}}(F)$ curves as a basis for making drift-diffusion calculations of the current-voltage $I(V)$ characteristics. These calculations involve self-consistent numerical solution of the Poisson equation and the current continuity equations, using a quasi-continuum model of the SL layers.

When $\theta=0^{\circ}$ (bottom curve in Fig. 4) and $90^{\circ}$ (top curve in Fig. 4), the $I(V)$ curves are knee shaped. At high voltages, the current remains high because the decrease in drift velocity that occurs at high electric fields is compensated by an increase in the number of conduction electrons in the miniband. Figure 4 shows that tilting the magnetic field modulates the knee with a shoulder and additional current peak, which originates from the $n=1$ resonant maximum 
in $v_{\mathrm{d}}(F)$. The resonant peak is weaker in the $I(V)$ curves than in the $v_{\mathrm{d}}(F)$ plots because the electric field is not uniform throughout the SL layers, and so the resonance condition is not satisfied near the emitter contact.

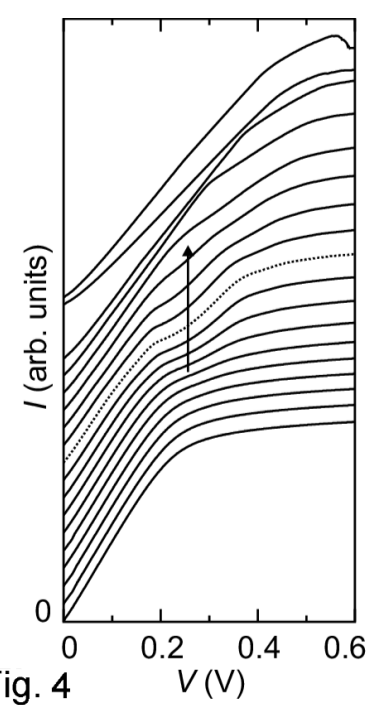

Fig. 4

Fig. 4. Current-voltage curves calculated for the SL with $B=11 \mathrm{~T}$, and $\theta=0^{\circ}$ (bottom trace) to $90^{\circ}$ (top trace) at $5^{\circ}$ intervals. Curve for $\theta=45^{\circ}$ is shown dotted. The knee in the curve for $\theta=0^{\circ}$ originates from the onset of the Bloch electron localisation. For tilt angles in the range $30^{\circ} \leq \theta \leq 55^{\circ}$, the knee is modulated by a shoulder (arrowed), followed by an increase in the gradient of the curve, which originates from delocalisation of the electron orbits due to the formation of stochastic webs in the electron phase space (see Fig. 2b).

Fig. 5. Conductance-voltage curves calculated for the SL with $B=11 \mathrm{~T}$, and $\theta=0^{\circ}$ (bottom trace) to $90^{\circ}$ (top trace) at $5^{\circ}$ intervals. Curve for $\theta=45^{\circ}$ is shown dotted. Conductance drop (arrow MB) is associated with the onset of the Bloch electron localisation. Stronger peaks labelled SW correspond to the arrowed feature in Fig. 4. They indicate a new type of resonance, which emerges as the magnetic field is tilted, and originates from delocalisation of the electron orbits due to the formation of stochastic webs in the electron phase space (see Fig. 2b).

To show the effect of stochastic web formation on the transport properties of the SL, it is useful to consider the first derivatives of these $I(V)$ curves, which are shown in Fig. 5. The lower trace in Fig. 5 shows the differential conductance $G=\mathrm{d} I / \mathrm{d} V$ calculated from the regular Bloch orbits at $\theta=0^{\circ}$. The sharp drop in conductance (arrow MB) corresponds to the knee in the corresponding $I(V)$ curve (Fig. 4) and originates from the onset of the Bloch electron localisation. This conductance drop persists as $\theta$ increases from $0^{\circ}$ to $90^{\circ}$. But the key feature 
of the $G(V)$ curves in Fig. 5 is the conductance peak (arrow SW), which emerges as theta increases from $0^{\circ}$.

In each $G(V)$ curve, the peak occurs near the voltage for which $\omega_{\mathrm{B}}=$ $\omega_{\mathrm{C}} \cos \theta$, which means that the phase space is threaded by an infinite stochastic web. The electrons undergo rapid diffusive motion through the web and through the SL itself, thereby generating the resonant peak in conductance. In real space, the electrons follow unbounded chaotic trajectories like that shown in Fig. 1b. The resonant conductance peak is strongest when $\theta$ is close to $45^{\circ}$ because, at this particular tilt angle, the plane wave that drives the electron orbits chaotic has maximal amplitude. Previous experimental studies of electron transport in SLs at $\theta=0^{\circ}$ have revealed the so-called Stark-cyclotron resonance, which occurs when the Wannier-Stark and Landau levels are equally spaced, that is when $\hbar \omega_{\mathrm{B}}=\hbar \omega_{\mathrm{C}}[25]$. This type of resonance is a purely quantum-mechanical effect because it requires energy-level quantisation [25]. We emphasise that the stronger conductance resonance that we predict in a tilted magnetic field has a fundamentally different origin from the Stark-cyclotron resonance and is a consequence of stochastic miniband transport rather than energy level quantisation.

\section{Summary}

Electrons in a SL miniband with a tilted magnetic field provide an experimentally accessible non-KAM chaotic system. In contrast to previous quantum-chaotic systems, the classical Hamiltonian originates from an intrinsically quantum-mechanical property of the SL: miniband dispersion. Despite involving only stationary electric and magnetic fields, this three-dimensional system is dynamically equivalent to a one-dimensional simple harmonic oscillator driven by a time-dependent plane wave so, in effect, the applied fields act like a $\mathrm{THz}$ source. When the cyclotron and Bloch frequencies are resonant, the phase space is threaded by an infinite stochastic web, which completely delocalises the electrons. This unique feature of non-KAM chaos produces a strong resonant peak in our theoretical conductance-voltage curves. In recent experiments on SLs with the same parameters as those considered here, we have observed the resonant enhancement of conductance predicted by our calculations [26]. Stochastic webs therefore have the potential to provide a highly sensitive and generic mechanism for controlling transport through periodic quantum and analogous photonic systems [27].

\section{References}

[1] For a review see H.-J. Stöckmann, Quantum Chaos - An Introduction, Cambridge University Press, Cambridge 2000.

[2] C.M. Marcus, A.J. Rimberg, R.M. Westervelt, P.F. Hopkins, A.C. Gossard, Phys. Rev. Lett. 69, 506 (1992).

[3] R. Fleischmann, T. Geisel, R. Ketzmerick, Phys. Rev. Lett. 68, 1367 (1992); D. Weiss, K. Richter, A. Menschig, R. Bergmann, H. Schweizer, K. von Klitzing, G. Weimann, Phys. Rev. Lett. 70, 4118 (1993). 
[4] A.M. Chang, H.U. Baranger, L.N. Pfeiffer, K.W. West, Phys. Rev. Lett. 73, 2111 (1994).

[5] Y.H. Zhang, J. Kastrup, R. Klann, K.H. Ploog, H.T. Grahn, Phys. Rev. Lett. 77, 3001 (1996).

[6] R.P. Taylor, R. Newbury, A.S. Sachrajda, Y. Feng, P.T. Coleridge, C. Dettmann, N. Zhu, H. Guo, A. Delage, P.J. Kelly, Z. Wasilewski, Phys. Rev. Lett. 78, 1952 (1997)

[7] A.S. Sachrajda, R. Ketzmerick, C. Gould, Y. Feng, P.J. Kelly, A. Delage, Z. Wasilewski, Phys. Rev. Lett. 80, 1948 (1998).

[8] J.P. Bird, R. Akis, D.K. Ferry, D. Vasileska, J. Cooper, Y. Aoyagi, T. Sugano, Phys. Rev. Lett. 82, 4691 (1999).

[9] Y. Takagaki, K.H. Ploog, Phys. Rev. B 61, 4457 (2000).

[10] T. Blomquist, I.V. Zozoulenko, Phys. Rev. B 64, 195301 (2001).

[11] W.E. Bies, L. Kaplan, M.R. Haggerty, E.J. Heller, Phys. Rev. E 63, 066214 (2001); W.E. Bies, L. Kaplan, E.J. Heller, Phys. Rev. E 64, 016204 (2001).

[12] A.P.S. de Moura, Y.-C. Lai, R. Akis, J.P. Bird, D.K. Ferry, Phys. Rev. Lett. 88, 236804 (2002).

[13] O.M. Bulashenko, L.L. Bonilla, Phys. Rev. B 52, 7849 (1995).

[14] K. Alekseev, G.P. Bermady, D.K. Campbell, E.H. Cannon, M.C. Cargo, Phys. Rev. B 54, 10625 (1996).

[15] E. Schöll, A. Wacker, in: Springer Proceedings in Physics, Vol. 79, Nonlinear Dynamics and Pattern Formation in Semiconductors and Devices, Ed. F.-J. Niedernestheide, Springer-Verlag, Berlin 1995, p. 21.

[16] T.M. Fromhold, L. Eaves, F.W. Sheard, M.L. Leadbeater, T.J. Foster, P.C. Main, Phys. Rev. Lett. 72, 2608 (1994); P.B. Wilkinson, F.W. Sheard, L. Eaves, J. Miao, G. Edwards, Phys. Rev. Lett. 75, 1142 (1995).

[17] D.L. Shepelyansky, A.D. Stone, Phys. Rev. Lett. 74, 2098 (1995).

[18] P.B. Wilkinson, T.M. Fromhold, L.Eaves, F.W. Sheard, N. Miura, T. Takamasu, Nature 380, 608 (1996).

[19] For a review see G.M. Zaslavsky, R.Z. Sagdeev, D.A. Usikov, A.A. Chemikov, Weak Chaos and Quasi-Regular Patterns, Cambridge University Press, Cambridge 1991.

[20] V.Ya. Demikhovskii, D.I. Kamenev, G.A. Luna-Acosta, Phys. Rev. B 52, 3351 (1995); Phys. Rev. B 59, 294 (1999).

[21] T.M. Fromhold, A.A. Krokhin, C.R. Tench, S. Bujkiewicz, P.B. Wilkinson, F.W. Sheard, L. Eaves, Phys. Rev. Lett. 87, 046803 (2001).

[22] A. Patanè, D. Sherwood, L. Eaves, T.M. Fromhold, M. Henini, P.C. Main, G. Hill, Appl. Phys. Lett. 81, 661 (2002).

[23] L. Esaki, R. Tsu, IBM J. Res. Dev. 14, 61 (1970).

[24] H.J. Hutchinson, A.W. Higgs, D.C. Herbert, G.W. Smith, J. Appl. Phys. 75, 320 (1994).

[25] L. Canali, M. Lazzarino, L. Sorba, F. Beltram, Phys. Rev. Lett. 76, 3618 (1996). 
[26] T.M. Fromhold, A. Patanè, S. Bujkiewicz, P.B. Wilkinson, D. Fowler, D. Shearwood, S.P. Stapleton, A.A. Krokhin, L. Eaves, M. Henini, N.S. Sankeshwar, F.W. Sheard, Nature 428, 726 (2004).

[27] P.B. Wilkinson, T.M. Fromhold, Opt. Lett. 28, 1034 (2003). 